\title{
The negative aftermath of prostate biopsy: prophylaxis, complications and antimicrobial stewardship: results of the global prevalence study of infections in urology 2010-2019
}

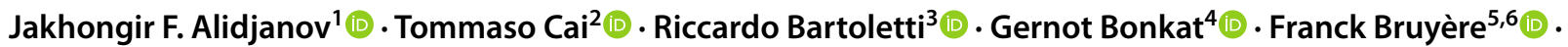

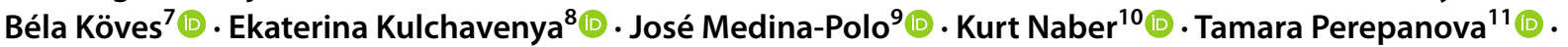

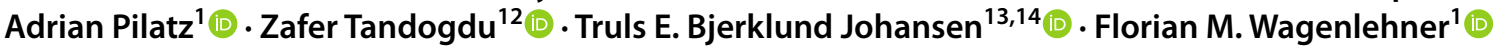

Received: 29 October 2020 / Accepted: 25 January 2021 / Published online: 22 February 2021

(c) The Author(s) 2021

\begin{abstract}
Purpose To evaluate and report the complications, and to analyse antimicrobial stewardship aspects following prostate biopsies (P-Bx) based on the data from a 9-year global study.

Methods The primary outcome was to compare complications after P-Bx between patients of two cohorts: 2010-2014 and 2016-2019. Primary outcomes included symptoms of lower and severe/systemic urinary tract infection (LUTIS and SUTIS, respectively), and positive urine culture. Readmission to hospital after P-Bx, need for additional antimicrobial therapy, consumption of different antimicrobial agents for prophylaxis and therapy were evaluated. Students $t$ test and chi-square test were used for comparative analyses.

Results Outcome data were available for 1615 men. Fluoroquinolones-based prophylaxis rate increased from $72.0 \%$ in 2010-2014 to $78.6 \%$ in 2015-2019. Overall rates of complications increased from 6 to $11.7 \%$ including an increase in symptomatic complications from 4.7 to $10.2 \%$, mainly due to an increase in LUTIS. Rates of patients seeking additional medical help in primary care after P-Bx increased from 7.4 to $14.4 \%$; cases requiring post P-Bx antibiotic treatment increased from 6.1 to $9.7 \%$, most of which received fluoroquinolones. Transperineal P-Bx was significantly associated with LUTIS. Following transrectal P-Bx, 2.8\% developed febrile infections and 4.0\% required hospitalisation. Two men (0.12\%) died after transrectal P-Bx due to sepsis.

Conclusions The rates of complications after P-Bx tended to increase in time, as well as rates of patients seeking additional medical help in the post-P-Bx period. To reduce the risk of infectious complications and to comply with the principles of antibiotic stewardship, clinicians should switch to the transperineal biopsy route.
\end{abstract}

Keywords Prostate biopsy $\cdot$ Antibiotic resistance $\cdot$ Prostate cancer $\cdot$ Antibiotics $\cdot$ Fluoroquinolones

\section{Introduction}

Prostate biopsy ( $\mathrm{P}-\mathrm{Bx})$ is one of the most commonly performed urological procedures worldwide, more than one million biopsies are performed annually in the United States

Jakhongir F. Alidjanov and Tommaso Cai shared first authorship.

Truls E. Bjerklund Johansen and Florian M. Wagenlehner shared last authorship.

Florian M. Wagenlehner

florian.wagenlehner@chiru.med.uni-giessen.de

Extended author information available on the last page of the article alone [1]. Since introduction of transrectal biopsies, the symptomatic infectious complications rates have varied from 1.9 to $27.7 \%$ and have tended to increase [2-7]. Apart from the contamination category of the procedure, risk factors for post-biopsy infections remain unclear. However, in a recent systematic review, Borghesi et al. reported that the highest rates of infective complications were related to comorbidities and old age [6].

Results of the Global Prevalence Study of Infections in Urology (GPIU), a cross-sectional survey, initiated in 2003 by the board of the European Society for Infections in Urology (ESIU) showed that antimicrobial resistance (AMR) among common uropathogens increasingly lead to failure of antibiotic prophylaxis, thereby underlining the importance 
of site-specific antimicrobial stewardship programmes [8-11]. A side study on infectious complications of P-Bx in 2010 and 2011 reported that $5.2 \%$ of men experienced symptomatic urinary tract infection (UTI), 3.5\% experienced febrile infectious complications and $3.1 \%$ required hospital re-admission after P-Bx [12].

The primary aim of the present study was to identify temporal trends in infective complications after P-Bx within the years 2010 to 2019 and compare two cohorts 2010-2014 and 2016-2019. Our secondary aim was to analyse antimicrobial stewardship aspects such as the percentage of patients seeking medical help of any kind and the rate of antibiotic treatment for infective complications after P-Bx.

\section{Methods}

\section{Study design and setting}

The GPIU P-Bx study is a prospective, observational online study, conducted annually in urology departments to audit the prevalence of infective complications after P-Bx across centres and countries and to evaluate factors associated with a higher risk of complications. Patients undergoing P-Bx within 2 weeks before the predefined study days each year were eligible for inclusion. Each enrolled patient was followed up for 2 weeks after the biopsy [12-14]. The severity of infectious complications was grouped according to the Center for Disease Control (CDC) criteria, in line with European Association of Urology (EAU) guidelines [15]. Ethical approval and regulatory issues were responsibility of each study centre [16].

Here, we cover the study years from 2010 to 2019, except for 2015 when the P-Bx study was not conducted due to updating of the online database. We, therefore, decided to use this year as a cut-off to allocate patients into two cohorts 2010-2014 and 2016-2019, respectively, depending on the year of registration.

\section{Patient information}

The clinical report forms consisted of two parts. The first part included items on:

a. Patient characteristics, such as age, use of antibiotics in the preceding 6 months, history of urinary tract infections (UTI) and history of antibiotic treatment in the preceding 6 months, prostate volume, prostate-specific antigen (PSA) value and previous P-Bx.

b. Biospsy characteristics such as biopsy route, results of preoperative urine culture; if preoperative bowel preparation was performed and its type; if antibiotic prophylaxis was administered and if yes, which antimicrobial agent, the number of biopsy cores taken and use of local anaesthesia.

The second part of the study report form included:

a. Clinical and microbiological outcome variables after the P-Bx such as presence and severity of symptoms of UTI at any time up to 2 weeks after P-Bx, (re)admission to hospital, results of post-P-Bx urine culture, type of antibiotics administered for treatment of infectious complications.

b. Histological parameters such as presence and grade of histopathologic inflammation (low, moderate, severe).

\section{Data processing and statistical analysis}

Symptoms were classified as lower UTI symptoms (LUTIS) such as frequency, dysuria, urgency and prostate pain, or symptoms of severe/systemic UTI (SUTIS), such as loin pain, rigour and fever. The majority of variables were categorical (presence/absence of symptom or characteristic) and, hence, dichotomized. Multiple imputations were performed on missing numerical values depending on the distribution, the median was used for the non-normally distributed and mean was used for normally distributed numerical variables. Missing categorical variables were not imputed to avoid biases.

Outcomes such as LUTIS, SUTIS and positive urine culture after P-Bx were assessed and compared both separately and in combination. Admission to hospital and need for antimicrobial treatment in the post-P-Bx period were considered as negative outcomes.

Continuous variables were presented in averages such as median and interquartile ranges (IQR) and compared between cohorts using two-sided Student $t$ test with the Welch correction in cases of inequality of variances. Categorical variables were presented in proportions of the total study population and compared with the chi-square test concerning the total numbers of cases in cohorts. Statistical significance was set at 0.05 . Statistical analysis and graphical representation of the results were performed using R-studio supporting the R-4.0.2 with in-built and additional packages [17-19].

\section{Results}

Data from 258 clinics from 55 countries were considered valid for analysis (see Appendix). The number of participating centres went down from 174 in years 2010-2014 to 84 in years 2016-2019 (Table 1). The number of inputted valid cases was 2215 . The range per study centre was $1-95$ (median $=5$, $\mathrm{IQR}=2-10$ ). Complete outcome data were available for 1615 
Table 1 Demographics and outcomes in patients according to the total sample and cohorts

\begin{tabular}{|c|c|c|c|c|}
\hline Parameter & Total & Cohort 2010-2014 & Cohort 2016-2019 & $P$ value* \\
\hline \multicolumn{5}{|l|}{ Demographics } \\
\hline Number of participating centres, $n$ & 258 & 174 & 84 & n.a \\
\hline Number of cases, $n(\%)$ & $1615(100.0)$ & $1204(74.6)$ & $411(25.4)$ & n.a \\
\hline Age, year, median (IQR) & $66(61.0-72.0)$ & $66(61.0-71.0)$ & $67(61.0-72.0)$ & 0.675 \\
\hline Volume of prostate, ml, median (IQR) & $46(43.0-86.5)$ & $46(46.0-161.0)$ & $46(35.0-60.0)$ & $<0.001$ \\
\hline PSA value, ng/ml, median (IQR) & $9.0(6.1-41.7)$ & $10.4(6.4-98.0)$ & $8.0(6.0-12.0)$ & $<0.001$ \\
\hline Route of biopsy: perineal/transrectal, $n(\%)$ & $56 / 1559(3.5 / 96.5)$ & $32 / 1172(2.7 / 97.3)$ & 24/387 (5.8/94.2) & 0.004 \\
\hline Number of cores, median (IQR) & $12(10.0-12.0)$ & $12(10.0-12.0)$ & $12(10.0-12.0)$ & 0.273 \\
\hline Repeated biopsy, $n(\%)$ & $307(19.0)$ & $218(18.1)$ & $89(21.7)$ & 0.131 \\
\hline Number of previous biopsies, median (IQR) & $1(1.0-2.0)$ & $1(1.0-2.0)$ & $1(1.0-2.0)$ & 0.458 \\
\hline History of UTIs, $n(\%)$ & $132(8.2)$ & $102(8.5)$ & $30(7.3)$ & 0.514 \\
\hline Comorbid diabetes, $n(\%)$ & $112(6.9)$ & $58(4.8)$ & $54(13.1)$ & 0.026 \\
\hline Presence of the urinary catheter, $n(\%)$ & $73(4.5)$ & $44(3.7)$ & $29(7.1)$ & 0.138 \\
\hline Duration of the catheter stay, days, median (IQR) & $10(5.0-20.0)$ & $10(7.0-20.0)$ & $6(1.0-24.0)$ & 0.544 \\
\hline $\mathrm{AB}$ therapy for any reason in preceding 6 months., $n(\%)$ & $203(12.6)$ & $138(11.5)$ & $65(15.8)$ & 0.051 \\
\hline With fluoroquinolones, $n(\%)$ & $114(7.1)$ & $75(6.2)$ & $39(9.5)$ & 0.055 \\
\hline With penicillins, $n(\%)$ & $39(2.4)$ & $27(2.2)$ & $12(2.9)$ & 0.636 \\
\hline With TMP-SMX, $n(\%)$ & $20(1.2)$ & $15(1.2)$ & $5(1.2)$ & 1.000 \\
\hline With cephalosporins, $n(\%)$ & $14(0.9)$ & $11(0.9)$ & $3(0.7)$ & 0.918 \\
\hline With other antimicrobial agents, $n(\%)$ & $16(1.0)$ & $10(0.8)$ & $6(1.5)$ & 0.410 \\
\hline $\begin{array}{l}\text { Duration of the } \mathrm{AB} \text { therapy in preceding } 6 \text { months, days, median } \\
\text { (IQR) }\end{array}$ & $7(7.0-14.0)$ & $7(5.0-10.5)$ & $7(7.0-14.0)$ & 0.027 \\
\hline Positive urine culture before $\mathrm{P}-\mathrm{Bx}, n(\%)$ & $27(1.7)$ & $17(1.4)$ & $10(2.4)$ & 0.645 \\
\hline $\mathrm{AB}$ prophylaxis before $\mathrm{P}-\mathrm{Bx}, n(\%)$ & $1503(93.1)$ & $1109(92.1)$ & $394(95.9)$ & 0.013 \\
\hline With fluoroquinolones, $n(\%)$ & $1190(73.7)$ & $867(72.0)$ & $323(78.6)$ & 0.011 \\
\hline With combinations of ABs, $n(\%)$ & $137(8.5)$ & $129(10.7)$ & $8(1.9)$ & $<0.001$ \\
\hline With cephalosporins, $n(\%)$ & $56(3.5)$ & $29(2.4)$ & $27(6.6)$ & $<0.001$ \\
\hline With aminoglycosides, $n(\%)$ & $52(3.2)$ & $31(2.6)$ & $21(5.1)$ & 0.019 \\
\hline With penicillins, $n(\%)$ & $17(1.1)$ & $10(0.8)$ & $7(1.7)$ & 0.224 \\
\hline With TMP-SMX, $n(\%)$ & $15(0.9)$ & $10(0.8)$ & $5(1.2)$ & 0.684 \\
\hline With oxacephems, $n(\%)$ & $15(0.9)$ & $15(1.2)$ & $0(0.0)$ & 0.048 \\
\hline With other antimicrobial agents, $n(\%)$ & $21(1.3)$ & $18(1.5)$ & $3(0.7)$ & 0.352 \\
\hline Duration of the $\mathrm{AB}$ prophylaxis, days, median (IQR) & $3(1.0-5.0)$ & $3(1.0-5.0)$ & $2(1.0-4.0)$ & 0.014 \\
\hline Bowel preparation before $\mathrm{P}-\mathrm{Bx}, n(\%)$ & $570(35.3)$ & $408(33.9)$ & $162(39.4)$ & 0.057 \\
\hline Enema, $n(\%)$ & $444(27.5)$ & $317(26.3)$ & $127(30.9)$ & 0.094 \\
\hline Lavage, $n(\%)$ & $32(2.0)$ & $24(2.0)$ & $8(1.9)$ & 1.000 \\
\hline Other, $n(\%)$ & $94(5.8)$ & $67(5.6)$ & $27(6.6)$ & 0.544 \\
\hline Anaesthesia, $n(\%)$ & $1099(68.0)$ & $801(66.5)$ & $298(72.5)$ & 0.055 \\
\hline Local, $n(\%)$ & $985(61.0)$ & $721(59.9)$ & $264(64.2)$ & 0.204 \\
\hline General, $n(\%)$ & $97(6.0)$ & $69(5.7)$ & $28(6.8)$ & 0.531 \\
\hline Spinal, $n(\%)$ & $17(1.1)$ & $11(0.9)$ & $6(1.5)$ & 0.525 \\
\hline \multicolumn{5}{|l|}{ Outcomes } \\
\hline Histopathologic signs of inflammation in the prostatic tissue, $n(\%)$ & $455(28.2)$ & $321(26.7)$ & $134(32.6)$ & 0.025 \\
\hline Mild, $n(\%)$ & $280(17.3)$ & $214(17.8)$ & $66(16.1)$ & 0.545 \\
\hline Moderate, $n(\%)$ & $141(8.7)$ & $83(6.9)$ & $58(14.1)$ & $<0.001$ \\
\hline Severe, $n(\%)$ & $34(2.1)$ & $24(2.0)$ & $10(2.4)$ & 0.736 \\
\hline Cases with at least 1 negative outcome, $n(\%)$ & $122(7.6)$ & $74(6.1)$ & $48(11.7)$ & $<0.001$ \\
\hline Symptomatic cases & $111(6.9)$ & $64(5.3)$ & $47(11.4)$ & $<0.001$ \\
\hline LUTIS, $n(\%)$ & $98(6.1)$ & $56(4.7)$ & $42(10.2)$ & $<0.001$ \\
\hline Dysuria, $n(\%)$ & $75(4.6)$ & $42(3.5)$ & $33(8.0)$ & $<0.001$ \\
\hline
\end{tabular}


Table 1 (continued)

\begin{tabular}{|c|c|c|c|c|}
\hline Parameter & Total & Cohort 2010-2014 & Cohort 2016-2019 & $P$ value* \\
\hline Frequency, $n(\%)$ & 47 (2.9) & $29(2.4)$ & $18(4.4)$ & 0.060 \\
\hline Urgency, $n(\%)$ & $38(2.4)$ & $24(2.0)$ & $14(3.4)$ & 0.149 \\
\hline Prostate pain, $n(\%)$ & $32(2.0)$ & $17(1.4)$ & $15(3.6)$ & 0.009 \\
\hline Number of LUTIS per case, median (IQR) & $2(1.0-3-0)$ & $2(1.0-3.0)$ & $1(1.0-3.0)$ & 0.651 \\
\hline Symptoms of UTI, $n(\%)$ & $46(2.8)$ & $33(2.7)$ & $13(3.2)$ & 0.785 \\
\hline Loin pain, $n(\%)$ & $4(0.2)$ & $3(0.2)$ & $1(0.2)$ & 1.000 \\
\hline Rigour, $n(\%)$ & $6(0.4)$ & $1(0.1)$ & $5(1.2)$ & 0.005 \\
\hline Fever, $n(\%)$ & $42(2.6)$ & $31(2.6)$ & $11(2.7)$ & 1.000 \\
\hline Number of UTI symptoms per case, median (IQR) & $1(1.0-1.0)$ & $1(1.0-1.0)$ & $1(1.0-2.0)$ & 0.025 \\
\hline Number of symptoms (LUTIS and UTI) per case, median (IQR) & $2(1.0-3.0)$ & $2(1.0-3.0)$ & $2(1.0-3.0)$ & 0.353 \\
\hline Positive urine culture after biopsy, $n(\%)$ & $39(2.4)$ & $28(2.3)$ & $11(2.7)$ & 1.000 \\
\hline Symptomatic cases with positive urine culture after biopsy, $n(\%)$ & $28(1.7)$ & $18(1.5)$ & $10(2.4)$ & 0.265 \\
\hline Isolated E. coli, $n(\%)$ & $29(1.8)$ & $20(1.7)$ & $9(2.2)$ & 0.577 \\
\hline Resistance to antimicrobials, $n(\%)$ & $103(6.4)$ & $51(4.2)$ & $52(12.7)$ & $<0.001$ \\
\hline Cephalosporins, $n(\%)$ & $25(1.5)$ & $12(1.0)$ & $13(3.2)$ & 0.030 \\
\hline Penicillins, $n(\%)$ & $19(1.2)$ & $8(0.7)$ & $11(2.7)$ & 0.059 \\
\hline Aminoglycosides, $n(\%)$ & $12(0.7)$ & $5(0.4)$ & $7(1.7)$ & 0.050 \\
\hline Fluoroquinolones, $n(\%)$ & $19(1.2)$ & $16(1.3)$ & $3(0.2)$ & 0.185 \\
\hline TMP-SMX, $n(\%)$ & $9(0.6)$ & $6(0.5)$ & $3(0.7)$ & 1.000 \\
\hline Other classes of antimicrobials, $n(\%)$ & $19(1.2)$ & $4(0.3)$ & $15(3.6)$ & $<0.001$ \\
\hline Patients, seeking for medical help after P-Bx, $n(\%)$ & $185(11.5)$ & $114(9.5)$ & $71(17.3)$ & $<0.001$ \\
\hline At primary care clinician's, $n(\%)$ & $148(9.2)$ & $89(7.4)$ & $59(14.4)$ & $<0.001$ \\
\hline At emergency room, $n(\%)$ & $37(2.3)$ & $25(2.1)$ & $12(2.9)$ & 0.426 \\
\hline Patients, required hospitalisation, $n(\%)$ & $60(3.7)$ & $46(3.8)$ & $14(3.4)$ & 0.816 \\
\hline To urology ward, $n(\%)$ & $52(3.2)$ & $41(3.4)$ & $11(2.7)$ & 0.575 \\
\hline To internal medicine ward, $n(\%)$ & $6(0.4)$ & $3(0.2)$ & $3(0.7)$ & 0.361 \\
\hline To intensive care ward, $n(\%)$ & $2(0.1)$ & $2(0.2)$ & $0(0.0)$ & 0.988 \\
\hline Antibacterial therapy, prescribed after P-Bx, $n(\%)$ & $113(7.0)$ & $73(6.1)$ & $40(9.7)$ & 0.016 \\
\hline With fluoroquinolones, $n(\%)$ & $54(3.3)$ & $33(2.7)$ & $21(5.1)$ & 0.032 \\
\hline With cephalosporins, $n(\%)$ & $23(1.4)$ & $15(1.2)$ & $15(1.9)$ & 0.427 \\
\hline With aminoglycosides, $n(\%)$ & $8(0.5)$ & $5(0.4)$ & $3(0.7)$ & 0.706 \\
\hline With penicillins, $n(\%)$ & $7(0.4)$ & $5(0.4)$ & $2(0.5)$ & 1.000 \\
\hline With combination of antimicrobial agents, $n(\%)$ & $7(0.4)$ & $5(0.4)$ & $2(0.5)$ & 1.000 \\
\hline With other antimicrobial agents, $n(\%)$ & $14(0.9)$ & $10(0.8)$ & $4(1.0)$ & 1.000 \\
\hline Duration of the antibacterial therapy, days, median (IQR) & $5(5.0-10.0)$ & $7(5.0-8.5)$ & $5(5.0-10.0)$ & 0.937 \\
\hline Resolved cases, $n(\%)$ & $1458(90.3)$ & $1114(95.0)$ & $314(76.4)$ & $<0.001$ \\
\hline Unresolved cases, $n(\%)$ & $66(4.1)$ & $53(4.4)$ & $13(3.2)$ & 0.342 \\
\hline Lethal cases, $n(\%)$ & $2(0.1)$ & $1(0.1)$ & $1(0.2)$ & 1.000 \\
\hline
\end{tabular}

*Cohort 2010-2014 vs. cohort 2016-2019

cases (72.9\% of total), all of which were included in further analysis. Of these, 1204 (74.6\%) were included in years
2010-2014, and 411 (25.4\%) in years 2016-2019 (Supplementary Fig. 1, Table 1). 


\section{Total population}

\section{Demographics}

Median (IQR) age of patients included in the analysis was 66 (61.0-72.0) years. Of them, 307 (19.0\%) underwent repeat biopsy. The median (IQR) number of previous biopsies was 1 (1.0-2.0).

A history of UTI was noted in $132(8.2 \%)$ patients, comorbid diabetes in $112(6.9 \%)$ and 73 (4.5\%) patients had a urinary catheter at time of biopsy.

A history of previous antimicrobial treatment was positive in 203 (12.6\%) patients, with fluoroquinolones being the most widely prescribed antimicrobial in 114 (7.1\% of total).

Transrectal P-Bx was performed in 1559 (96.5\%) patients and 56 (3.5\%) underwent transperineal P-Bx.

Positive urine culture before $\mathrm{P}-\mathrm{Bx}$ was noted in 27 (1.7\%) cases (Table 1). Antimicrobial prophylaxis before P-Bx was reported in 1503 (93.1\%) patients, with fluoroquinolones as the most frequently prescribed class in $1190(73.7 \%)$ (Table 1).

\section{Clinical outcomes}

LUTIS were recorded in 98 (6.1\%) patients, of which the most common single symptom was dysuria which was observed in 75 (4.6\%) patients, and SUTIS was noted in 46 (2.8\%), of which 42 cases (2.6\%) had fever (Fig. 1a).

The proportion of cases with LUTIS was significantly $(p<0.001)$ higher in the group of patients who underwent perineal P-Bx (17.9\%) than in those, who underwent transrectal P-Bx (5.6\%). The proportion of cases with SUTIS was $3.0 \%$ among patients who underwent transrectal P-Bx. No case of SUTIS was recorded among the patients after perineal P-Bx.
Antimicrobial treatment was prescribed to 113 (7.0\%) patients after P-Bx. Fever was the single symptom that most often led to treatment with antimicrobial agents $(88.1 \%)$, followed by prostate pain $(71.9 \%)$ and rigour (66.7\%) (Supplementary Fig. 2). 185 (11.5\%) patients were seeking additional medical help, and 60 patients (3.7\%) were hospitalised due to complications (Fig. 1b). All hospitalised cases belonged to patients who underwent transrectal P-Bx.

In $66(4.1 \%)$ patient's complications had not resolved within the 2 week follow-up after P-Bx, and $2(0.1 \%)$ lethal cases were noted due to urosepsis after transrectal P-Bx (Table 1).

\section{Microbiological outcomes}

Thirty-nine of 103 tested cases $(2.4 \%$ of total) had a positive urine culture after P-Bx (Table 1, Fig. 1a). All cases of positive urine culture were recorded in the group of patients who underwent transrectal P-Bx. A single uropathogen was found in $36(2.2 \%)$ cases, and a mixed flora was found in 3 cases $(0.2 \%)$. Escherichia coli was the most commonly isolated uropathogen and was noted in 29 $(1.8 \%)$ cases, followed by Enterococci $(n=3)$ and coagulase-negative Staphylococci $(n=3)(0.2 \%$ per case/sample) of cases, Enterobacter spp. $(n=2)$, and Klebsiella spp. $(n=2)(0.1 \%$ per case/sample). Proteus and Pseudomonas species were found in two cases $(<0.1 \%$ per case/sample). The highest resistance rates of uropathogens were found against cephalosporins $(n=25)$, penicillins $(n=19)$, fluoroquinolones $(n=19)$, and aminoglycosides $(n=12)$.
A

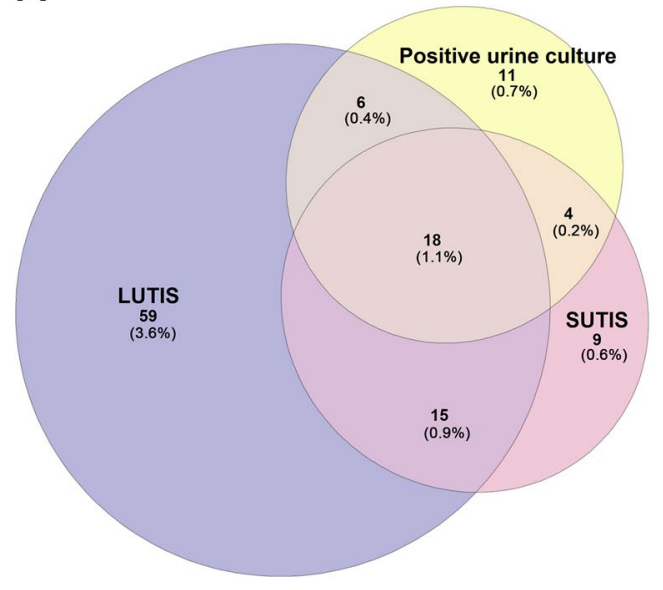

B

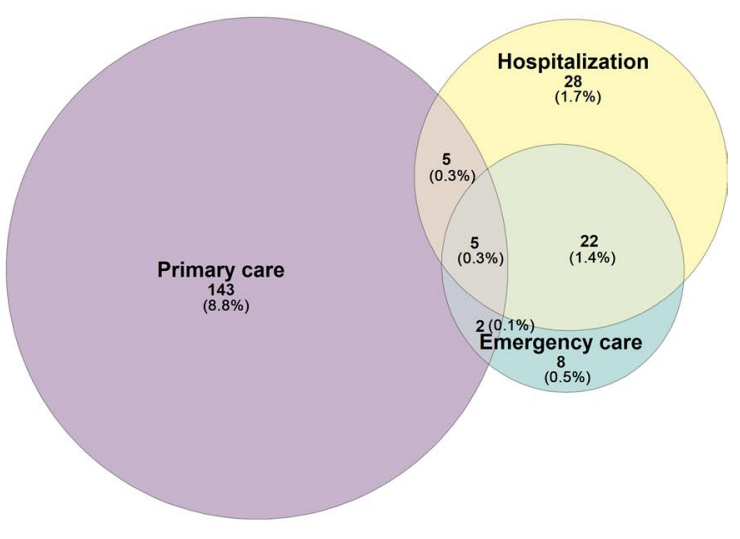

Fig. 1 a Proportions of negative outcomes after P-Bx. b Proportions of cases requiring medical assistance after P-Bx 


\section{Comparison between cohorts and trends over time}

\section{Demographics}

The average age of the patients did not differ significantly between cohorts, neither did the proportions of repeat biopsy, history of UTI and antimicrobial treatment, positive urine culture before biopsy, presence of urinary catheter or duration of catheterisation. The proportion of patients with diabetes mellitus was significantly higher in cohort 2016-2019 (Table 1).

\section{Biopsy-related variables}

The preparation and performance of P-Bx (bowel preparation, anaesthesia, number of biopsy cores) did also not differ. However, the route of P-Bx showed a statistically significant increase in the rates of transperineal $\mathrm{P}-\mathrm{BX}$ with a subsequent significant decrease in the number of transrectal P-Bx with 32 and 1172 (2.7 and 97.3\%) cases in 2010-2014 vs 24 and 387 (5.8 and 94.2\%) cases in 2016-2019 for transperineal and transrectal routes, respectively $(p<0.001)$.

\section{Symptoms}

Overall rates of complications increased from $6.1 \%$ in the cohort of 2010-2014 to $11.7 \%$ in the 2016-2019 cohort $(p<0.001)$ (Supplementary Fig. 1, Table 1).

The proportion of LUTIS, dysuria and prostate pain was significantly higher in the cohort of 2016-2019 $(p<0.01)$. The overall rates of SUTIS remained the same between cohorts; while, the rates of rigour as single symptom were significantly higher in the cohort of 2016-2019, as well as the average number of SUTIS $(p<0.05)$.

The proportions of cases which resolved within 2 weeks after P-Bx decreased significantly from 95.0 to $76.4 \%$ from the period 2010-2014 to 2016-2019 ( $p<0.001)$ (Table 1).

\section{Microbiological variables}

Among patients with a history of antibiotic treatment before $\mathrm{P}-\mathrm{Bx}$, the duration of treatment was significantly longer in the cohort 2016-2019 $(p=0.027)$. The proportion of cases receiving antimicrobial prophylaxis (especially with fluoroquinolones, cephalosporins, and aminoglycosides) was significantly higher in the cohort 2016-2019; whereas, average duration of prophylaxis was significantly lower $(p<0.05)$ (Table 1$)$.
The resistance of uropathogens was significantly higher in the cohort 2016-2019 particularly due to resistance against cephalosporins $(p<0.05)$ (Table 1$)$.

\section{Need for medical assistance}

The proportions of patients seeking medical help within 2 weeks after P-Bx increased in 2016-19, mainly among patients who sought their GP`s office $(p<0.001)$; whereas, the numbers of patients who needed hospitalisation did not differ significantly between cohorts $(p=0.816)$. Prescription of antimicrobial therapy after P-Bx increased from 6.1 to $9.7 \%$, with a significant increase in the prescription of fluoroquinolones $(p<0.05)$.

\section{Discussion}

Infectious complications occur in 5-7\% of all transrectal $\mathrm{P}-\mathrm{Bx}$. Severe infections requiring hospitalisation are seen after $1-3 \%$ of biopsies and fatal events are reported in $0.1-1.3 \%$ [20]. During the recent years, there has been a rise in infectious complications after P-Bx, thought to be due to increasing antibiotic resistance, especially to fluoroquinolones which have been recommended by the European and the American Association of Urology Guidelines as the first-choice antibiotic prophylaxis in $\mathrm{P}-\mathrm{Bx}$ in the past $[15$, 21]. The antibiotic stewardship perspectives of the extensive use of fluoroquinolones have been questioned [1,21].

In this study, we could demonstrate a significant global increase in the rate of infective complications after transrectal P-Bx from 2010 to 2019. We found a twofold increase in the rate of symptoms from 5.3\% in the cohort of 2010-2014 to $11.4 \%$ in the cohort of 2016-2019, paralleled by an increase in the rate of dysuria, prostate pain (LUTIS), rigour (a symptom of SUTIS) and an average number of symptoms per case. Moreover, there were two cases of death due to septic shock after transrectal P-Bx (0.1\%), a rate that equals house mortality after radical prostatectomy [22]. The number of patients who sought medical help for any complication and the number of patients who received antibiotic treatment for infective complications increased significantly in the most recent cohort. Also, the number of resolved cases within two weeks decreased from $95.0 \%$ in 2010-2014 to $76.4 \%$ in $2016-2019$.

The use of fluoroquinolones as prophylactic agents increased during study years and remain the most commonly prescribed agents for $\mathrm{P}-\mathrm{Bx}$ prophylaxis worldwide. In the first analysis of the GPIU P-Bx study, fluoroquinolones were used for prophylaxis in $98.2 \%$ of patients and $60 \%$ of all bacterial strains isolated after the procedure were resistant to this drug [12]. Several authors have shown that E. coli is the most common pathogen in terms of infective complications 
after P-Bx [23]. Moreover, prebiopsy rectal cultures have demonstrated a fluoroquinolone-resistant colonisation rate of $10-22 \%$ [20]. We did not find a significant difference in rates of fluoroquinolone-resistant strains between cohorts, but there was an almost threefold increase in overall resistance rates of uropathogens against antimicrobial agents from 4.2 to $12.7 \%$. We also found a significant increase in the overall prescription of antimicrobial prophylaxis for almost all classes of antimicrobial agents, including fluoroquinolones and cephalosporins ( 72.0 vs. 78.6 , and 2.4 vs. $6.6 \%$ for 2010-2014 vs. 2016-2019, respectively). Patients with a history of preceding antimicrobial treatment were at higher risk of developing complications after transrectal P-Bx. Steensels and co-authors demonstrated that the use of fluoroquinolones 6 months before $\mathrm{P}-\mathrm{Bx}$ was associated with an increased risk of faecal carriage of fluoroquinolone-resistant E. coli strains [24]. Our findings demonstrate that the rate of symptomatic infective complications after transrectal P-Bx is high and is associated with antimicrobial resistance and use of antibiotics both as prophylaxis and as treatment of complications. This violates the principles of antimicrobial stewardship and increases health care costs.

Scott et al. showed that in cases of empirical antimicrobial prophylaxis without prior urine culture, the infection rate was 3.4\% (95\% CI 2.6-4.3\%). In cases of culture-based targeted antibiotic prophylaxis, the infection rate was $0.8 \%$ (95\% CI 0.4-1.3\%) [23]. In our first GPIU P-Bx study, we argued that the rate of systemic infection was higher than earlier reports due to GPIU recruiting patients from a global average of urology departments and not from selected centres only. Other recent publications also demonstrate an increase in negative outcomes after P-Bx, including infectious complications [25-27]. In this study, we included all symptomatic complications, not only infections confirmed by microbiological culture. In the GPIU protocol, we do request microbiological analysis, but this was performed in only 103 cases (6.4\%). By separating symptoms to SUTIS and LUTIS, respectively, we could demonstrate that SUTIS such as loin pain, rigour and fever were associated with transrectal P-Bx and not with transperineal P-Bx. The transrectal biopsy was associated with almost all remaining predefined complications (outcomes), such as positive urine culture after P-Bx and need for hospitalisation within the 2 weeks after P-Bx. Moreover, both lethal cases due to septic shock occurred after transrectal P-Bx. Our analysis demonstrated that patients who underwent transrectal $\mathrm{P}-\mathrm{Bx}$ more often developed SUTIS, while patients with transperineal P-BX were more likely to develop prostate pain and LUTIS.

Several clinical factors are associated with a higher risk of symptomatic complications [1]. In the present study, we demonstrate that transrectal P-Bx itself is a risk factor for complications and need for hospitalisation; while, the transperineal route is associated with LUTIS only. We believe this underlines that the contamination category of the biopsy procedure is the most significant risk factor for infective complications.

Fluoroquinolones, which have remained the most commonly used drugs in urological practice, were recently suspended for P-Bx prophylaxis by the European Medical Agency (EMA) [28]. Alternative antibiotic regimens are, therefore, warranted. A meta-analysis including only randomised studies exhibited limited evidence for using aminoglycosides, cephalosporins and fosfomycin trometamol for transrectal P-Bx prophylaxis [27]. Our findings support this view. We could, however, show that the use of aminoglycosides as prophylaxis was associated with a higher risk of symptomatic complications and a higher need for antibiotic therapy in the follow-up period after transrectal P-Bx. Detailed knowledge of local resistance data must, therefore, be taken into account in antibiotic prophylaxis protocols [29]. Our data suggest that to reduce patients' risk of infectious complications and to comply with the principles of antibiotic stewardship, transperineal P-Bx should be prioritised [26, 30].

\section{Strengths and limitations}

The worldwide, multicentric, multinational and prospective design are strengths of this study. The long enrolment period allows for detecting changes in the rates of infective complications and bacterial resistance over time.

Limitations to consider are the few centres per country, which means that data are not representative for each country. The low number of microbiological cultures after the procedure might be considered a study limitation. We argue, however, that our study reports the real-life situation in all centres.

\section{Conclusions}

We emphasise the worldwide increase in complication rates (up to $11.4 \%$ ) and the average number of symptoms such as dysuria, prostate pain and rigour after P-Bx, traceable from 2010 to 2019 in the GPIU study. The rate of fluoroquinolones prescribed as prophylaxis before, and as antimicrobial treatment after P-Bx also increased significantly. Transperineal P-Bx itself was a risk factor for development of LUTIS; whereas, transrectal P-Bx was associated with a wider spectrum of post-P-Bx complications including LUTIS and SUTIS, positive urine culture after the biopsy and risk of hospitalisation. The most severe infectious complications like febrile UTI and mortality were only seen after transrectal P-Bx. 
Supplementary Information The online version contains supplementary material available at https://doi.org/10.1007/s00345-021-03614-8.

Author contributions TEBJ, KN, GB, ZT, FMW developed the study plan, designed and planned the study. Authors listed in (Appendix) contributed to patient recruitment and study data entry. JA processed the study data and performed the statistical analysis. BK, JMP, AP, ZT contributed to interpretation of the data. GB, TC, TEBJ, and FMW wrote the first draft of the manuscript. BK, RB, GB, FB, KN, TEBJ contributed for writing, reviewing and editing manuscript and validation of data. GB, FB, EK, TP, EK, AP contributed in critical revision of the prefinal version of the manuscript. JA, FMW and TEBJ updated, complemented and edited manuscript which was reviewed, discussed, finalised and approved by all authors.

Funding Open Access funding enabled and organized by Projekt DEAL.

\section{Compliance with ethical standards}

Conflict of interest All authors declare the following conflict of interests: all authors are members of the European Section of Infection in Urology (ESIU) of the European Association of Urology (EAU). The GPIU study is organised by the board of the European Section of Infections in Urology (ESIU) and endorsed by the EAU and the EAU Research Foundation (EAU RF). The study is performed in collaboration with the Asian Association of UTI and STI (AAUS), the International Society of Antimicrobial Chemotherapy (ISAC), and the Interregional Association of Clinical Microbiology and Antimicrobial Chemotherapy. The study platform is maintained by the Technische Hochschule Mittelhessen (THM), Germany and sponsored by Merian Iselin Foundation, Switzerland. Otherwise the authors did not receive support from any organisation for the submitted work. No funding was received to assist with the preparation of this manuscript. We thank all the GPIU Investigators who provided essential information (listed in Appendix).

Ethical approval The study was approved by the leading ethics committee (Ethik Kommission am Fachbereich Medizin of the Justus Liebig University Giessen, Germany. Ethical vote: AZ.: 116/07). Local ethical approval and regulatory issues were responsibility of each study centre. We certify that the study was performed in accordance with the ethical standards as laid down in the 1964 declaration of Helsinki and its later amendments.

Informed consent Participants data are imputed entirely anonymized. Informed consent was therefore waived by the ethical commission.

Open Access This article is licensed under a Creative Commons Attribution 4.0 International License, which permits use, sharing, adaptation, distribution and reproduction in any medium or format, as long as you give appropriate credit to the original author(s) and the source, provide a link to the Creative Commons licence, and indicate if changes were made. The images or other third party material in this article are included in the article's Creative Commons licence, unless indicated otherwise in a credit line to the material. If material is not included in the article's Creative Commons licence and your intended use is not permitted by statutory regulation or exceeds the permitted use, you will need to obtain permission directly from the copyright holder. To view a copy of this licence, visit http://creativecommons.org/licenses/by/4.0/.

\section{References}

1. Loeb S, Carter HB, Berndt SI, Ricker W, Schaeffer EM (2011) Complications after prostate biopsy: data from SEER-Medicare. J Urol 186(5):1830-1834. https://doi.org/10.1016/j. juro.2011.06.057

2. Wendel RG, Evans AT (1967) Complications of punch biopsy of the prostate gland. J Urol 97(1):122-126. https://doi.org/10.1016/ s0022-5347(17)62995-1

3. Davison P, Malament M (1971) Urinary contamination as a result of transrectal biopsy of the prostate. J Urol 105(4):545-546. https ://doi.org/10.1016/s0022-5347(17)61571-4

4. Fawcett DP, Eykyn S, Bultitude MI (1975) Urinary tract infection following transrectal biopsy of the prostate. Br J Urol 47(6):679681. https://doi.org/10.1111/j.1464-410x.1975.tb04036.x

5. Loeb S, Vellekoop A, Ahmed HU, Catto J, Emberton M, Nam R, Rosario DJ, Scattoni V, Lotan Y (2013) Systematic review of complications of prostate biopsy. Eur Urol 64(6):876-892. https ://doi.org/10.1016/j.eururo.2013.05.049

6. Borghesi M, Ahmed H, Nam R, Schaeffer E, Schiavina R, Taneja S, Weidner W, Loeb S (2017) Complications after systematic, random, and image-guided prostate biopsy. Eur Urol 71(3):353-365. https://doi.org/10.1016/j.eururo.2016.08.004

7. Cheng KC, Lam WC, Chan HC, Ngo CC, Cheung MH, So HS, Lam KM (2019) Emergency attendances and hospitalisations for complications after transrectal ultrasound-guided prostate biopsies: a five-year retrospective multicentre study. Hong Kong Med J 25(5):349-355. https://doi.org/10.12809/hkmj197825

8. Johansen TE, Cek M, Naber KG, Stratchounski L, Svendsen MV, Tenke P, Pep, investigators PE-s, Board of the European Society of Infections in Urology (2006) Hospital acquired urinary tract infections in urology departments: pathogens, susceptibility and use of antibiotics. Data from the PEP and PEAP-studies. Int J Antimicrob Agents 28(Suppl 1):S91-107. https://doi.org/10.1016/j.ijant imicag.2006.05.005

9. Cek M, Tandogdu Z, Wagenlehner F, Tenke P, Naber K, Bjerklund-Johansen TE (2014) Healthcare-associated urinary tract infections in hospitalized urological patients-a global perspective: results from the GPIU studies 2003-2010. World J Urol 32(6):1587-1594. https://doi.org/10.1007/s00345-013-1218-9

10. Tandogdu Z, Kakariadis ETA, Naber K, Wagenlehner F, Bjerklund Johansen TE (2019) Appropriate empiric antibiotic choices in health care associated urinary tract infections in urology departments in Europe from 2006 to 2015: a Bayesian analytical approach applied in a surveillance study. PLoS ONE 14(4):e0214710. https://doi.org/10.1371/journal.pone.0214710

11. Koves B, Tenke P, Tandogdu Z, Cai T, Bogenhard F, Wullt B, Naber K, Bartoletti R, Cek M, Kulchavenya E, Perepanova T, Pilatz A, Bonkat G, Erik Bjerklund Johansen T, Wagenlehner F (2019) Transurethral resection of the prostate: are we following the guidelines? - outcomes from the global prevalence of infections in urology (GPIU) study. J Chemother 31(1):15-22. https:// doi.org/10.1080/1120009X.2018.1542552

12. Wagenlehner FM, van Oostrum E, Tenke P, Tandogdu Z, Cek M, Grabe M, Wullt B, Pickard R, Naber KG, Pilatz A, Weidner W, Bjerklund-Johansen TE, Investigators G (2013) Infective complications after prostate biopsy: outcome of the Global Prevalence Study of Infections in Urology (GPIU) 2010 and 2011, a prospective multinational multicentre prostate biopsy study. Eur Urol 63(3):521-527. https://doi.org/10.1016/j.eururo.2012.06.003

13. Tandogdu Z, Bartoletti R, Cai T, Cek M, Grabe M, Kulchavenya E, Koves B, Menon V, Naber K, Perepanova T, Tenke P, Wullt B, 
Johansen TE, Wagenlehner F (2016) Antimicrobial resistance in urosepsis: outcomes from the multinational, multicenter Global Prevalence of Infections In Urology (GPIU) Study 2003-2013. World J Urol 34(8):1193-1200. https://doi.org/10.1007/s0034 5-015-1722-1

14. Wagenlehner F, Tandogdu Z, Bartoletti R, Cai T, Cek M, Kulchavenya E, Koves B, Naber K, Perepanova T, Tenke P, Wullt B, Bogenhard F, Bjerklund Johansen TE, Investigators G (2016) The Global Prevalence Of Infections In Urology (GPUI) Study: a worldwide surveillance study in urology patients. Eur Urol Focus 2(4):345-347. https://doi.org/10.1016/j.euf.2016.03.004

15. Bonkat GC, Bartoletti R, Bruyère $F$, Cai $T$, Geerlings SE, Köves B, Schubert S, Wagenlehner F, Associates: G, Mezei T, Pilatz A, Pradere B, Veeratterapillay R (2020) EAU Guidelines on Urological Infections. In: EAU Guidelines. Edn, presented at the EAU Annual Congress Amsterdam, EAU Guidelines Office, Arnhem, The Netherlands

16. Wagenlehner F, Tandogdu Z, Bartoletti R, Cai T, Cek M, Kulchavenya E, Koves B, Naber K, Perepanova T, Tenke P, Wullt B, Bogenhard F, Johansen TE (2016) The Global Prevalence of Infections in Urology Study: a long-term, worldwide surveillance study on urological infections. Pathogens 5(1):10. https://doi. org/10.3390/pathogens5010010

17. The R Development Core Team (2017) R: a language and environment for statistical computing. CRAN, Vienna

18. Wickham H (2017) tidyverse: easily install and load the 'Tidyverse'

19. Larsson J (2020) eulerr: area-proportional Euler and venn diagrams with ellipses

20. Fontana M, Boeri L, Montanari E (2018) Update on techniques to prevent infections associated with prostate needle biopsy. Curr Opin Urol 28(4):392-397. https://doi.org/10.1097/MOU.00000 00000000507

21. Wolf JS Jr, Bennett CJ, Dmochowski RR, Hollenbeck BK, Pearle MS, Schaeffer AJ (2008) Urologic surgery antimicrobial prophylaxis best practice policy panel: best practice policy statement on urologic surgery antimicrobial prophylaxis. J Urol 179(4):13791390. https://doi.org/10.1016/j.juro.2008.01.068

22. Preisser F, Mazzone E, Nazzani S, Bandini M, Tian Z, Marchioni M, Steuber T, Saad F, Montorsi F, Shariat SF, Huland H, Graefen M, Tilki D, Karakiewicz PI (2018) Comparison of perioperative outcomes between cytoreductive radical prostatectomy and radical prostatectomy for nonmetastatic prostate cancer. Eur Urol 74(6):693-696. https://doi.org/10.1016/j.eururo.2018.07.006

23. Scott S, Harris PN, Williamson DA, Liss MA, Doi SAR, Roberts MJ (2018) The effectiveness of targeted relative to empiric prophylaxis on infectious complications after transrectal ultrasound-guided prostate biopsy: a meta-analysis. World J Urol 36(7):1007-1017. https://doi.org/10.1007/s00345-018-2217-7

24. Steensels D, Slabbaert K, De Wever L, Vermeersch P, Van Poppel $\mathrm{H}$, Verhaegen J (2012) Fluoroquinolone-resistant $E$. coli in intestinal flora of patients undergoing transrectal ultrasound-guided prostate biopsy should we reassess our practices for antibiotic prophylaxis? Clin Microbiol Infect 18(6):575-581. https://doi.org /10.1111/j.1469-0691.2011.03638.x

25. Knaapila J, Gunell M, Syvanen K, Ettala O, Kahkonen E, Lamminen T, Seppanen M, Jambor I, Rannikko A, Riikonen J, Munukka E, Eerola E, Hakanen AJ, Bostrom PJ (2019) Prevalence of complications leading to a health care contact after transrectal prostate biopsies: a prospective, controlled, multicenter study based on a selected study cohort. Eur Urol Focus 5(3):443-448. https://doi.org/10.1016/j.euf.2017.12.001

26. Grummet J, Gorin MA, Popert R, O’Brien T, Lamb AD, Hadaschik B, Radtke JP, Wagenlehner F, Baco E, Moore CM, Emberton M, George AK, Davis JW, Szabo RJ, Buckley R, Loblaw A, Allaway M, Kastner C, Briers E, Royce PL, Frydenberg M, Murphy DG, Woo HH (2020) "TREXIT 2020": why the time to abandon transrectal prostate biopsy starts now. Prostate Cancer Prostatic Dis 23(1):62-65. https://doi.org/10.1038/s41391-020-0204-8

27. Pilatz A, Dimitropoulos K, Veeratterapillay R, Yuan Y, Omar MI, MacLennan S, Cai T, Bruyère F, Bartoletti R, Köves B, Wagenlehner F, Bonkat G, Pradere B (2020) Antibiotic prophylaxis for the prevention of infectious complications following prostate biopsy: a systematic review and meta-analysis. J Urol 204(2):224-230

28. Bonkat G, Pilatz A, Wagenlehner F (2019) Time to adapt our practice? the European commission has restricted the use of fluoroquinolones since march 2019. Eur Urol 76(3):273-275. https:// doi.org/10.1016/j.eururo.2019.06.011

29. Cai T, Verze P, Bartoletti R, Mirone V, Johansen TEB (2015) Infectious complications after prostate biopsy: time to rethink our clinical practice. WJCU World J Clin Urol 4(2):78

30. Pilatz A, Veeratterapillay R, Dimitropoulos K, Omar MI, Pradere B, Yuan Y, Cai T, Mezei T, Devlies W, Bruyère F, Bartoletti R, Köves B, Geerlings S, Schubert S, Grummet J, Mottet N, Wagenlehner F, Bonkat G (2021) European association of urology position paper on the prevention of infectious complications following prostate biopsy. Eur Urol 79(1):11-15

Publisher's Note Springer Nature remains neutral with regard to jurisdictional claims in published maps and institutional affiliations.

\section{Authors and Affiliations}

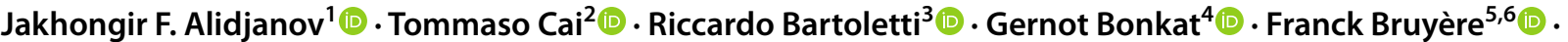

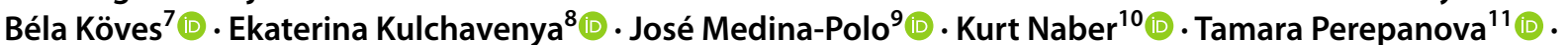

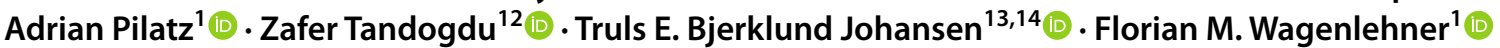

1 Clinic for Urology, Pediatric Urology and Andrology, JustusLiebig-University Giessen, Giessen, Germany

2 Department of Urology, Santa Chiara Hospital, Trento, Italy

3 Department of Urology, University of Pisa, Pisa, Italy

4 Department of Urology, Alta Uro AG, Basel, Switzerland

5 Urologie, CHU Bretonneau, Tours, France
6 Université François Rabelais de Tours, PRES Centre Val de Loire, Tours, France

7 Department of Urology, South-Pest Teaching Hospital, Budapest, Hungary

8 Urogenital Department, Novosibirsk Research TB Institute, Koves Str 1. 1204, Budapest, 630040 Novosibirsk, Russian Federation 
9 Department of Urology, Hospital Universitario 12 de Octubre, Madrid, Spain

10 School of Medicine, Technical University of Munich, Munich, Germany

11 Department of Urinary Tract Infections and Clinical Pharmacology N.A, Lopatkin Scientific Research Institute of Urology and Interventional Radiology, Branch of the National Medical Research Radiological Centre of the Ministry of Health of the Russian Federation, Moscow, Russian Federation
12 Department of Urology, University College London Hospitals, London, UK

13 Department of Urology, Oslo University Hospital, Institute of Clinical Medicine, University of Oslo, Oslo, Norway

14 Institute of Clinical Medicine, University of Aarhus, Aarhus, Denmark 